\title{
Entameba histolytica as a cause of diarrhea in hemodialysis patients in Qena
}

\section{Yaser A. Fahmi ${ }^{\text {, }}$ Mohammed E.Merghany ${ }^{\text {,Abd Elkader A.Hashim }}$, Asmaa M. El- $\operatorname{Kady}^{\mathbf{a}}$}

a Department of Medical Parasitology, Faculty of Medicine, South Valley University, Qena, Egypt.

${ }^{b}$ Medical Parasitology Department, Faculty of Medicine, Assuit University, Assiut, Egypt.

'Internal Medicine Department, Faculty of Medicine, South Valley University, Qena, Egypt.

\section{Abstract:}

Background: Chronic Kidney Disease (CKD) is a globally increasing condition that almost always finally leads to renal replacement therapy. Intestinal parasitic infections are amongst the widest spread of all chronic human infections worldwide.

Materials and methods: 50 stool samples were collected from patients who suffer from Chronic Kidney Disease from Qena Governorate Hospitals and 50 stool samples from healthy control group. The fecal samples were examined macroscopically to determine the consistency, presence of blood or mucus and presence of parasites.

Results: Intestinal protozoal infections were detected in 33 out of 50 patients in the CKD group (66\%). Entamebahistolytica infection was detected in 7 out of 50 patients in the CKD group representing $14 \%$ of the total infections.

Conclusions: Entamoebahistolyticainfection in the CKD are within the universal levels.

Keywords: Entamoebahistolytica, hemodialysis, Qena

\section{INTRODUCTION}

Chronic Kidney Disease (CKD) is a globally increasing condition that almost always finally leads to renal replacement therapy e.g. (dialysis treatment). CKD is also a state of immunodeficiency with increased susceptibility to infections that are the second most common cause of death after vascular diseases among dialysis patients (Rocco et al., 2002, Collins et al., 2006, Inaguma et al ., 2008).

Major risk factors for development and progression of CKD include diabetes, hypertension and old age. Nearly $45 \%$ of incident kidney failure is attributed to diabetes and another $20 \%$ is attributed to chronic hypertension. Other less common but important causes includes analgesic 
abuse, primary glomerulonephritis, lupus, and polycystic kidney disease. Notably, diabetes and hypertension are also important risk factors for cardiovascular disease and, to some extent, influence the high incidence of cardiovascular disease in the CKD population (Weiner et al., 2006).

CKD results in disruption of the intestinal barrier structure and marked alteration of its microbial flora -events that play a major role in the pathogenesis of inflammation and uremic toxicity (Vaziri et al., 2012).

The fact that patients with Chronic Kidney Disease (CKD) have a disorder in their immune system was first noticed byDammin et al. (1957). The CKD has negative impacts on neutrophil chemotaxis, phagocytosis, and bactericidal actions and $\mathrm{T}$ cell function. Patients with a suppressed immune system due to $\mathrm{CKD}$ catch parasitic infections more easily, when the infection risks and related complications of these patients, who are open to infections, are considered, studies towards preventing infections are very important (Karadag et al., 2013).

Gastrointestinal infections are more common and more severe in immunocompromised individuals. Intestinal parasitic infections are amongst the widest spread of all chronic human infections worldwide. Opportunistic infections occur in patients with impaired host defences and are caused by infectious agents that do not ordinarily produce disease in healthy individuals. Among the parasites, that cause gastrointestinal infections in the immunocompromised patient population, are the Cryptosporidium species, Isospora, Microsporidia, Giardia lamblia, Entamoebahistolytica and Strongyloidesstercoralis (Ankuret al., 2013).

\section{Materials and Methods:}

\section{Study Design:}

In the present study, after approval from the scientific ethics committee and obtaining an informed written consent, 50 stool samples were collected from patients who suffer from Chronic Kidney Disease from Qena Governorate Hospitals and 50 stool samples from healthy control group who don't suffer from Chronic Kidney Disease from outpatient clinics from June to December 2017.

Each collected sample was divided into two parts; $1^{\text {st }}$ part for early microscopic examination, the $2^{\text {nd }}$ part for preservation and late investigations.

Samples were collected in clean tight fitting container and every sample was labelled properly with the patient's name and date of collection

A portion of each faecal sample was added to formalin $10 \%$ in a vial to give a $3: 1$ ratio of preservative (formalin 10\%) to fecal material.

The fecal samples were examined macroscopically to determine the consistency, presence of blood or mucus and presence of parasites 
An amount of each faecalsample was mixed with one drop of $0.85 \%$ Nacl. A coverslip ( 22 by $22 \mathrm{~mm}$ ) was placed on the suspension then it is examined under the low power objective of microscope and the power was increased gradually to the oil immersion lens examination.

Smear 1 to 2 drops of specimen on the slide, and allow it to air dry. Fix with absolute methanol for $1 \mathrm{~min}$.Flood slide with Kinyoun'scarbolfuchsin, and stain for 5 min then Rinse slide briefly ( 3 to 5 s) with $50 \%$ ethanol. Rinse thoroughly with water then decolorize with $1 \%$ sulfuric acid for $2 \mathrm{~min}$ or until no more color runs from the slide. Rinse slide with water and drain it. Counterstain with methylene blue or brilliant green for $1 \mathrm{~min}$ then rinse slide with water and allow it to dry. Examine using low or high dry objectives.

\section{Statistical analysis:}

Data were organized, tabulated, and statistically analyzed using SPSS version, 16.00. For quantitative data, mean and standard deviation were calculated. Chi-square test $\left(\chi^{2}\right)$ was used to compare the frequency data.

$\mathrm{P}<0.05$ indicates significant values.

$\mathrm{P}<0.001$ indicates highly significant values.

\section{Results}

This study was conducted on 50 patients with Chronic Kidney Disease from Qena Governorate Hospitals and 50 healthy control individuals. They were 31 males (62\%) and 19 females (18\%) in the CKD patients group with age ranging from 38 to 73 years The number of Patients from urban areas was 23 compared to 27 patients from rural areas. Intestinal protozoal infections were detected in 33 out of 50 patients in the CKD group $(66 \%)$. Entamebahistolytica infection was detected in 7 out of 50 patients in the CKD group representing $14 \%$ of the total infections. As regard age groups in the CKD group, Entamoebahistolytica infection was not found in patients $<40$ years ( $0 \%$ of age group), in 1 of 7 patients from 40 to 50 years ( $14.29 \%$ of age group), in 3 of 24 patients from 50 to 60 years (12.50\% of age group), in 3 of 18 patients $>60$ years $(16.67 \%$ of age group). Thus, the age $>60$ years was the highest affected age group withEntamebahistolytica infection.

\section{Discussion:}

Immunocompromised patients are more vulnerable to parasitic infections (Emami et al., 2011). Patients with chronic renal failure, who are on hemodialysis, resulting in dysfunction of the immune response, are also prone to a variety of opportunistic infections( Azami et al., 2010).

In the present study, stool samples were collected and examined from 50 patients with chronic kidney disease and 50 healthy controls, we recognized that there was a high prevalence of intestinal protozoal infections in hemodialysis patients $(66 \%)$ compared to $(26 \%)$ in the healthy control group. Entamebahistolytica infection was detected in 7 patients with prevalence rate $14 \%$ in hemodialysis patients. Which is consistent with the results 
obtained by Fredrico et al., (2013), and Yousry et al., (2015) who found that the prevalence of Entamoebahistolytica infection was $3.6 \%$ among 110 hemodialysis patients and $2 \%$ in chronic kidney disease patients and $2 \%$ in the healthy controls in Saudi Arabia respectively. Similar results were obtained in Iran by Omrani et al., (2015) who found that the prevalence of Entamoeba histolytica infection was 2.5 $\%$ among 85 hemodialysis patients. Karadag et al., (2013)found similar results where the prevalence of Entamoebahistolytica infection was $2.1 \%$ among 142 hemodialysis patients in Turkey.

On the other hand, in another study done by El nadi and Taha (2004) in Sohag, , Egyptthe prevalence of Entamoebahistolytica infection was $56 \%$ among 50 hemodialysis patients which is much higher than our results and may be explained by the predominance of Entamoebahistolytica in normal individuals in Sohag.

\section{References:}

Ankur Kashyap1, Mahendra Pal Singh, Madhu and UjjalaGhoshal, (2013): Occurrence of Gastrointestinal Opportunistic Parasites in Immunocompromised Patients in Northern India. Journal of Biology, 1: 7780.

Azami M, Sharifi M, Hejazi SH, Tazhibi M.(2010): Intestinal parasitic infections in renal transplant recipients. Braz J Infect Dis, 2010; 14:15-18.
Collins, a.j., Kasiske, Herzog, Chavers and Gilbertson, (2006): United States Renal Data System 2005Annual Report.Am J Kidney Dis, 47:128-131.

Dammin, G. J., Couch, N. P. and Murray, J. E. (1957):Prolonged survival of skin homografts in uremic patients. "Ann N Y AcadSci, 64(5): 967-976.

El NadiN. andTaha A. (2004):Intestinal parasites detected amonghaemodialysis patients in Sohag University hospitals.EL-MINIA MED., BULL.,15(2):233-240.

\section{EmamiNaiini A, Shokrian A, Shahrzad} S, Aazami M, Hejazi SH, TazhibiM.(2011): The prevalence of intestinal parasitic and fungal agents in hemodialysis patients in Isfahan. $J$ Isfahan asiticMed Sch, 121:1655-1667.

Frederico F. GIL., Maxlene J. Barro, Nazaré A. Macedo, Carmelino G.E., et al.,(2013): Prevalence of intestinal parasitism and associated symptomatology among hemodialysis patients. Rev. Inst. Med. Trop. Sao.Paulo.,55(2):69-74.

Gh. Barzgarpour, S. Mehravar, F. Memari, F.Hajialiani, Z. Joneidi (2015): Prevalence of intestinal parasite infections and associated clinical symptoms among patients with end-stage renal disease Infect Dis, 14:15-18.

Inaguma, D., Nagaya, H., Hara, K., Tatemastu, M., Shinjo, H., Suzuki, S., Mishima, T andKurata, K. (2008): Relationship between serum 1, 25Dihydroxyvitamin D and mortality in 
patients with pre-dialysis chronic kidney disease. NephrolExpClin, 12(2): 126-31.

KaradagG., Gulden S., Tamer and ErkanDervisoglu.(2013): Investigation of intestinalparasitesin dialysis patients.Saudi Med J,34(7):714-718.

Rocco M. V., Yan, G., Gassman J., Lewis J. B., Ornt D., Weiss B. and Levey A. S.(2002). Comparison of causes of death using HEMO study and HCFA end stage renal disease death notification classification systems. The National Institutes of Health-funded Hemodialysis. Health Care Financing Administration " Am J Kidney Dis, 39(1): $146-53$.

V. FallahOmrani, Sh. Fallahi, A. Rostami, A. Siyadatpanah, Vaziri N.D., Dure-Smith B., Miller R., et.al., (2012). Pathology of gastrointestinal tract in chronic hemodialysis patients: an autopsy study of 78 cases. Am J Gastroenterol, 80:608-611.

Vaziri N. D., Ni, Z., Oveisi F., et al. (2002): Enhanced nitric oxide inactivation and protein nitration by reactive oxygen species in chronic renal insufficiency .Hypertension, 39: 135-41.

Weiner DE, Tabatabai S, Tighiouart H, Elsayed E, Bansal N, Griffith J, et al. (2006). Cardiovascular outcomes and all-cause mortality: exploring the interaction between CKD and cardiovascular disease. Am J Kidney Dis, 48(3):392-401.

Yousry A. Hawash, Laila Sh. Dorgham, El-Amir M. Amir, Osama F.
Sharaf, (2015): Prevalence of Intestinal Protozoa among Saudi Patients with Chronic Renal Failure : A Case-Control Study", Journal of Tropical Medicine, 2015:1-

9. https://doi.org/10.1155/2015/563478. 